\title{
Different Approaches to Estimation of the Gompertz Distribution under the Progressive Type-II Censoring Scheme
}

\author{
Kyeongjun Lee ${ }^{1}$ and Jung-In Seo $\mathbb{D}^{2}$ \\ ${ }^{1}$ Division of Mathematics and Big Data Science, Daegu University, No. 201, Daegudae-ro, Gyeongsan-si, Republic of Korea \\ ${ }^{2}$ Division of Convergence Education, Halla University, No. 28, Halladae-gil, Wonju-si, Republic of Korea \\ Correspondence should be addressed to Jung-In Seo; leehoo1928@gmail.com
}

Received 31 October 2019; Revised 8 July 2020; Accepted 30 July 2020; Published 7 September 2020

Academic Editor: Alessandro De Gregorio

Copyright (C 2020 Kyeongjun Lee and Jung-In Seo. This is an open access article distributed under the Creative Commons Attribution License, which permits unrestricted use, distribution, and reproduction in any medium, provided the original work is properly cited.

\begin{abstract}
This paper provides an estimation method for an unknown parameter by extending weighted least-squared and pivot-based methods to the Gompertz distribution with the shape and scale parameters under the progressive Type-II censoring scheme, which induces a consistent estimator and an unbiased estimator of the scale parameter. In addition, a way to deal with a nuisance parameter is provided in the pivot-based approach. For evaluation and comparison, the Monte Carlo simulations are conducted, and real data are analyzed.
\end{abstract}

\section{Introduction}

The probability density function (PDF) and cumulative distribution function $(\mathrm{CDF})$ of the random variable $X$ with the Gompertz distribution are, respectively, given by

$$
\begin{aligned}
& f(x ; \lambda, \theta)=\theta e^{\lambda x-(\theta / \lambda)\left(e^{\lambda x}-1\right)}, \\
& F(x ; \lambda, \theta)=1-e^{-(\theta / \lambda)\left(e^{\lambda x}-1\right)}, \quad x>0, \lambda, \theta>0,
\end{aligned}
$$

where $\lambda$ is the shape parameter and $\theta$ is the scale parameter. This distribution was first introduced by Gompertz [1] and often used to model human mortality. For this distribution, some studies have been done on the inference method based on the pivotal quantity to estimate confidence intervals (CIs) of the parameters of interest more efficiently. Chen [2] provided exact CIs under the Type-II censoring scheme. $\mathrm{Wu}$ et al. [3] provided exact CIs under the progressive Type-II censoring scheme. Studies of various estimation methods for this distribution have also been conducted by several researchers. Dey et al. [4] proposed different methods to estimate the PDF and CDF and compared the estimation methods based on the Monte Carlo simulations. Dey et al. [5] provided various mathematical and statistical properties and compared different estimation methods from both frequentist and Bayesian point of view. Moala and Dey [6] provided Bayesian analysis methods under the objective and subjective priors including Jeffreys prior, maximal data information prior, Singpurwalla's prior, and elicited prior.

In addition to this distribution, inferences based on the pivotal quantity have been studied for many distributions because the pivotal-based approach provides exact CIs even for small samples, as well as more efficient estimators than the maximum likelihood estimators (MLEs) in terms of bias. Wu [7] studied on a bathtub-shaped lifetime distribution under the progressive Type-II censoring scheme. Wang et al. [8] provided a generalized pivot-based method based on the progressive Type-II censored data from the reverse hazard distributions. Under the same censoring scheme, Seo and Kang $[9,10]$ provided closed forms of exact CIs for the scale parameter for each with and without the nuisance parameter such as the location parameter in a half logistic distribution.

Recently, a new estimation method based on a weighted regression framework has been proposed under some censoring schemes. Lu and Tao [11] applied the method based on a regression framework to the Pareto distribution where there is no censoring. Seo et al. [12] extended the idea 
of Lu and Tao [11] to the progressive Type-II censoring scheme to estimate unknown parameters of the Pareto distribution. Seo et al. [13] provided closed forms for unknown parameters of a two-parameter Rayleigh distribution using the same approach under the progressive Type-II censoring scheme.

This paper focuses on point estimation using the weighted regression framework and pivot-based methods based on the progressive Type-II censored data from the Gompertz distribution with the PDF (1). The remainder of the paper is structured as follows. Section 2 provides the weighted regression framework and pivot-based estimation methods corresponding to the progressive Type-II censored data from the Gompertz distribution. Section 3 evaluates and validates the proposed method through the Monte Carlo simulations and analyzes real data for purposes of illustration. Section 4 concludes the paper.

\section{Estimation}

This section gives a brief description for the progressive Type-II censoring scheme that is the generalization of the Type-II censoring scheme and that is one of the most popular censoring schemes and provides different approaches on estimation for unknown parameters of the Gompertz distribution with the PDF (1). For brevity's sake, the following notations are used throughout this paper:

Exp (1): the standard exponential distribution $\chi_{v}^{2}$ : the chi-squared distribution with $v$ degrees of freedom

$\operatorname{IG}(\alpha, \beta)$ : the inverse gamma distribution with the shape parameter $\alpha$ and the scale parameter $\beta$

$U(0,1)$ : the uniform distribution on the interval $(0,1)$

By Balakrishnan and Aggarwala [14], the progressive Type-II censoring scheme is described as follows. Let $m$ be the number of failures and $\mathscr{R}=\left(R_{1}, \ldots, R_{m}\right)$ denotes the censoring scheme, where $R_{i}$ is the number of removals at the $i$ th censoring time. In addition, suppose that the number of failures $m$ and the censoring scheme $\mathscr{R}=\left(R_{1}, \ldots, R_{m}\right)$ are fixed in advance. At the occurrence of the first failure, $R_{1}$ units are randomly withdrawn (or censored) from the $n-1$ surviving units. Subsequently, following the second observed failure, $R_{2}$ units are randomly censored from the $n-$ $2-R_{1}$ surviving units, and the process continues in this way until the $m-1$ th failure is observed. Finally, at the time of the $m$ th observed failure, all remaining $R_{m}=n-m-R_{1}-$ $\cdots-R_{m-1}$ units are censored from the test. As mentioned earlier, this scheme includes as a special case the conventional Type-II right censoring scenario that is defined when $R_{1}=\cdots=R_{m-1}=0, R_{m}=n-m$. In addition, the scenarios $m=n$ and $R_{1}=\cdots=R_{m}=0$ induce the complete sample situation.

Suppose that $X_{1: m: n}<\cdots<X_{m: m: n}$ is a progressive TypeII censored sample with the censoring scheme $\mathscr{R}$ from the Gompertz distribution. Then, the corresponding likelihood function is given by

$$
L(\lambda, \theta) \propto \prod_{i=1}^{m} f\left(x_{i: m: n} ; \lambda, \theta\right)\left[1-F\left(x_{i: m: n} ; \lambda, \theta\right)\right]^{R_{i}}=\theta^{m} e^{-\theta h(\lambda)+\lambda \sum_{i=1}^{m} x_{i: m: n}}
$$

where

$$
h(\lambda)=\frac{1}{\lambda} \sum_{i=1}^{m}\left(R_{i}+1\right)\left(e^{\lambda x_{i: m: n}}-1\right) .
$$

The MLEs $\hat{\lambda}$ and $\hat{\theta}$ can be found by maximizing the logarithm of the likelihood function (2) for $\lambda$ and $\theta$. The following provides approaches based on the regression framework and pivotal quantity which lead to a consistent estimator and an unbiased estimator of $\theta$ for known $\lambda$, respectively.

Let

$$
\begin{aligned}
Y_{i: m: n} & =-\log \left[1-F\left(x_{i: m: n} ; \lambda, \theta\right)\right] \\
& =\frac{\theta}{\lambda}\left(e^{\lambda X_{i: m: n}}-1\right), \quad i=1, \ldots, m .
\end{aligned}
$$

Then, $Y_{1: m: n}<\cdots<Y_{m: m: n}$ are progressive Type-II censored order statistics from $\operatorname{Exp}(1)$ with the mean

$$
E\left(Y_{i: m: n}\right)=\sum_{j=1}^{i}\left[\sum_{k=j}^{m}\left(1+R_{k}\right)\right]^{-1}
$$

and the variance

$$
\operatorname{Var}\left(Y_{i: m: n}\right)=\sum_{j=1}^{i}\left[\sum_{k=j}^{m}\left(1+R_{k}\right)\right]^{-2}, \quad 1 \leq i \leq m,
$$

given by Theorem 7.2.1 by Balakrishnan and Cramer [15]. From that fact, consider the following linear regression model:

$$
Y_{i: m: n}=E\left(Y_{i: m: n}\right)+\xi_{i}, \quad i=1, \ldots, m
$$

where $\xi_{i}$ is the error term with the mean 0 . For known $\lambda$, the regression model (7) provides a least-square estimator of $\theta$ as

$$
\widehat{\theta}_{l}(\lambda)=\frac{\lambda \sum_{i=1}^{m} E\left(Y_{i: m: n}\right)\left(e^{\lambda X_{i: m: n}}-1\right)}{\sum_{i=1}^{m}\left(e^{\lambda X_{i: m: n}}-1\right)^{2}} .
$$

By minimizing the following quantity for $\theta$ :

$$
\sum_{i=1}^{m}\left[\mathrm{E}\left(Y_{i: m: n}\right)-\frac{\theta}{\lambda}\left(e^{\lambda X_{i: m: n}}-1\right)\right]^{2} \text {. }
$$


However, the approach gives same weight on each point, and it is not proper because the variances of $Y_{i: m: n}(i=1, \ldots, m)$ do not satisfy the condition of being constant. As an alternative, $\mathrm{Lu}$ and Tao [11] considered weights that are inversely proportional to the corresponding variances. That is, a point with a low variance will be given a higher weight and a point with a higher variance will be given a low weight. Here, the weights corresponding to the progressive Type-II censoring scheme are defined as $w_{i}=1 / \operatorname{Var}\left(Y_{i: m: n}\right), i=1, \ldots, m$. Then, an estimator of $\theta$ based on the weighted regression framework is given by

$$
\widehat{\theta}_{w l}(\lambda)=\frac{\lambda \sum_{i=1}^{m} E\left(Y_{i: m: n}\right)\left(e^{\lambda X_{i: m: n}}-1\right) / \operatorname{Var}\left(Y_{i: m: n}\right)}{\sum_{i=1}^{m}\left(e^{\lambda X_{i: m: n}}-1\right)^{2} / \operatorname{Var}\left(Y_{i: m: n}\right)} .
$$

By minimizing the following quantity with the weighted square term for $\theta$ :

$$
\sum_{i=1}^{m} w_{i: m: n}\left[E\left(Y_{i: m: n}\right)-\frac{\theta}{\lambda}\left(e^{\lambda X_{i: m: n}}-1\right)\right]^{2} .
$$

Theorem 2.1. For known $\lambda$, the weighted square-least estimator $\widehat{\theta}_{w l}(\lambda)$ is a consistent estimator.

Proof. Let

$$
Z_{1 i: m: n}=\frac{Y_{i: m: n} E\left(Y_{i: m: n}\right)-E^{2}\left(Y_{i: m: n}\right)}{\operatorname{Var}\left(Y_{i: m: n}\right)}
$$

which implies convergence in probability [16]. In addition, $\sum_{i=1}^{m} Z_{3 i: m: n} / m^{2}$ does not converge to 0 as $m \longrightarrow \infty$ by the following inequality:

$$
\begin{aligned}
& E\left(\left|\frac{1}{m^{2}} \sum_{i=1}^{m} Z_{1 i: m: n}\right|\right)=0, \\
& E\left(\left|\frac{1}{m^{2}} \sum_{i=1}^{m} Z_{2 i: m: n}\right|\right)=\frac{1}{m},
\end{aligned}
$$

Here, both $Z_{1 i: m: n}$ and $Z_{2 i: m: n}$ converge in the mean to 0

Then, the estimator $\widehat{\theta}_{w l}(\lambda)$ can be written as

$$
\begin{aligned}
\hat{\theta}_{w l}(\lambda) & =\theta \frac{\sum_{i=1}^{m} Y_{i: m: n} E\left(Y_{i: m: n}\right) / \operatorname{Var}\left(Y_{i: m: n}\right)}{\sum_{i=1}^{m} Y_{i: m: n}^{2} / \operatorname{Var}\left(Y_{i: m: n}\right)} \\
& =\theta \frac{\sum_{i=1}^{m} Z_{1 i: m: n} / m^{2}+\sum_{i=1}^{m} Z_{3 i: m: n} / m^{2}}{\sum_{i=1}^{m} Z_{2 i: m: n} / m^{2}+\sum_{i=1}^{m} Z_{3 i: m: n} / m^{2}} .
\end{aligned}
$$

$$
\begin{aligned}
\frac{1}{m^{2}} \sum_{i=1}^{m} Z_{3 i: m: n} & =\frac{1}{m^{2}} \sum_{i=1}^{m} \frac{\left\{\sum_{j=1}^{i}\left[\sum_{k=j}^{m}\left(1+R_{k}\right)\right]^{-1}\right\}^{2}}{\sum_{j=1}^{i}\left[\sum_{k=j}^{m}\left(1+R_{k}\right)\right]^{-2}} \geq \frac{1}{m^{2}} \sum_{i=1}^{m} \frac{\left[\sum_{j=1}^{i}\left(m-1+\sum_{k=j}^{m} R_{k}\right)^{-1}\right]^{2}}{\sum_{j=1}^{i}(m-i+1)^{-2}}=\frac{1}{m^{2}} \sum_{i=1}^{m} i\left(\frac{m-i+1}{m-1+\sum_{k=j}^{m} R_{k}}\right)^{2} \\
& =\frac{1}{m^{2}\left(m-1+\sum_{k=j}^{m} R_{k}\right)^{2}}\left[\left(m^{2}+2 m\right) \sum_{j=1}^{m} j+\sum_{j=1}^{m} j^{3}-2(m+1) \sum_{j=1}^{m} j^{2}\right]
\end{aligned}
$$


TABLE 1: MSEs(biases) for $\lambda$ and $\theta$.

\begin{tabular}{|c|c|c|c|c|c|c|c|c|c|c|}
\hline$\lambda$ & $\theta$ & $n$ & $m$ & Scheme & $\widehat{\lambda}$ & $\hat{\lambda}_{p}$ & $\hat{\lambda}_{w l}$ & $\widehat{\theta}(\widehat{\lambda})$ & $\widehat{\theta}_{p}\left(\widehat{\lambda}_{p}\right)$ & $\widehat{\theta}_{w l}\left(\widehat{\lambda}_{w l}\right)$ \\
\hline \multirow[t]{27}{*}{0.1} & 0.5 & 20 & 20 & & $0.052(0.098)$ & $0.032(0.029)$ & $0.061(-0.035)$ & $0.033(-0.020)$ & $0.023(-0.003)$ & $0.060(0.059)$ \\
\hline & & & & I & $0.104(0.138)$ & $0.056(0.062)$ & $0.129(-0.033)$ & $0.040(-0.025)$ & $0.024(-0.020)$ & $0.079(0.064)$ \\
\hline & & & & II & $0.061(0.113)$ & $0.037(0.039)$ & $0.076(-0.033)$ & $0.034(-0.027)$ & $0.025(-0.010)$ & $0.070(0.060)$ \\
\hline & & & 18 & III & $0.082(0.125)$ & $0.047(0.052)$ & $0.100(-0.030)$ & $0.038(-0.026)$ & $0.024(-0.016)$ & $0.074(0.061)$ \\
\hline & & & & IV & $0.066(0.119)$ & $0.040(0.043)$ & $0.084(-0.035)$ & $0.033(-0.026)$ & $0.024(-0.011)$ & $0.069(0.060)$ \\
\hline & & & & I & $0.380(0.274)$ & $0.179(0.145)$ & $0.529(-0.070)$ & $0.062(-0.036)$ & $0.031(-0.040)$ & $0.150(0.096)$ \\
\hline & & & & II & $0.095(0.150)$ & $0.054(0.054)$ & $0.109(-0.027)$ & $0.046(-0.034)$ & $0.032(-0.015)$ & $0.099(0.068)$ \\
\hline & & & 14 & III & $0.223(0.214)$ & $0.113(0.103)$ & $0.268(-0.036)$ & $0.057(-0.034)$ & $0.031(-0.032)$ & $0.120(0.081)$ \\
\hline & & & & IV & $0.123(0.173)$ & $0.074(0.070)$ & $0.163(-0.042)$ & $0.042(-0.030)$ & $0.030(-0.019)$ & $0.095(0.070)$ \\
\hline & & 30 & 30 & & $0.029(0.068)$ & $0.023(0.027)$ & $0.031(-0.016)$ & $0.021(-0.020)$ & $0.018(-0.008)$ & $0.029(0.029)$ \\
\hline & & & & I & $0.073(0.100)$ & $0.045(0.056)$ & $0.081(-0.011)$ & $0.029(-0.018)$ & $0.019(-0.019)$ & $0.041(0.036)$ \\
\hline & & & & II & $0.035(0.077)$ & $0.025(0.029)$ & $0.037(-0.017)$ & $0.024(-0.020)$ & $0.020(-0.007)$ & $0.035(0.034)$ \\
\hline & & & 26 & III & $0.054(0.089)$ & $0.035(0.043)$ & $0.058(-0.011)$ & $0.027(-0.018)$ & $0.020(-0.015)$ & $0.039(0.034)$ \\
\hline & & & & IV & $0.039(0.083)$ & $0.028(0.033)$ & $0.042(-0.019)$ & $0.023(-0.019)$ & $0.019(-0.009)$ & $0.034(0.033)$ \\
\hline & & & & I & $0.394(0.246)$ & $0.181(0.158)$ & $0.555(-0.077)$ & $0.048(-0.025)$ & $0.024(-0.040)$ & $0.103(0.078)$ \\
\hline & & & 18 & II & $0.060(0.111)$ & $0.037(0.039)$ & $0.073(-0.027)$ & $0.034(-0.025)$ & $0.024(-0.009)$ & $0.066(0.055)$ \\
\hline & & & 18 & III & $0.202(0.181)$ & $0.100(0.103)$ & $0.266(-0.041)$ & $0.044(-0.024)$ & $0.023(-0.030)$ & $0.088(0.067)$ \\
\hline & & & & IV & $0.083(0.134)$ & $0.054(0.057)$ & $0.119(-0.040)$ & $0.029(-0.020)$ & $0.022(-0.014)$ & $0.062(0.054)$ \\
\hline & & 50 & 50 & & $0.013(0.039)$ & $0.012(0.008)$ & $0.017(-0.016)$ & $0.012(-0.012)$ & $0.012(0.001)$ & $0.017(0.020)$ \\
\hline & & & & $\mathrm{I}$ & $0.044(0.066)$ & $0.031(0.042)$ & $0.053(-0.002)$ & $0.018(-0.015)$ & $0.013(-0.017)$ & $0.023(0.017)$ \\
\hline & & & (4) & II & $0.018(0.049)$ & $0.016(0.017)$ & $0.021(-0.012)$ & $0.015(-0.016)$ & $0.014(-0.005)$ & $0.019(0.018)$ \\
\hline & & & 42 & III & $0.031(0.058)$ & $0.024(0.030)$ & $0.036(-0.003)$ & $0.017(-0.015)$ & $0.013(-0.012)$ & $0.022(0.016)$ \\
\hline & & & & IV & $0.024(0.058)$ & $0.023(0.029)$ & $0.030(-0.015)$ & $0.012(-0.011)$ & $0.012(-0.009)$ & $0.016(0.014)$ \\
\hline & & & & I & $0.355(0.201)$ & $0.180(0.167)$ & $0.435(-0.034)$ & $0.035(-0.019)$ & $0.019(-0.038)$ & $0.051(0.043)$ \\
\hline & & & & II & $0.035(0.077)$ & $0.025(0.029)$ & $0.037(-0.015)$ & $0.024(-0.019)$ & $0.020(-0.007)$ & $0.035(0.033)$ \\
\hline & & & 26 & III & $0.164(0.140)$ & $0.090(0.102)$ & $0.194(-0.014)$ & $0.032(-0.017)$ & $0.018(-0.030)$ & $0.045(0.038)$ \\
\hline & & & & IV & $0.049(0.093)$ & $0.039(0.046)$ & $0.059(-0.022)$ & $0.020(-0.013)$ & $0.017(-0.012)$ & $0.029(0.028)$ \\
\hline
\end{tabular}

Lemma 2.2. A quantity

$$
Q_{2}(\lambda)=2 \sum_{i=1}^{m-1} \log \left\{\frac{\sum_{j=1}^{m}\left(R_{j}+1\right)\left(e^{\lambda x_{j: m: n}}-1\right)}{\sum_{j=1}^{i}\left(R_{j}+1\right)\left(e^{\lambda x_{j: m: n}}-1\right)+\left[n-\sum_{j=1}^{i}\left(R_{j}+1\right)\right]\left(e^{\lambda x_{i: m: n}}-1\right)}\right\},
$$

has $\chi_{2(m-1)}^{2}$.

$$
S_{i}=\sum_{j=1}^{i}\left(R_{j}+1\right) Y_{j: m: n}+\left[n-\sum_{j=1}^{i}\left(R_{j}+1\right)\right] Y_{i: m: n}=\frac{\theta}{\lambda} \sum_{j=1}^{i}\left(R_{j}+1\right)\left(e^{\lambda X_{j: m: n}}-1\right)+\frac{\theta}{\lambda}\left[n-\sum_{j=1}^{i}\left(R_{j}+1\right)\right]\left(e^{\lambda X_{i: m: n}}-1\right), i=1, \ldots, m,
$$

and it induces the pivotal quantity $U_{(i)}=S_{i} /\left(S_{m}(i=1\right.$, $\ldots, m-1))$ that is order statistics from $U(0,1)$. Then, from the fact that $-2 \log U_{(i)}$ has $\chi_{2}^{2}$, the following quantity:

$$
Q_{2}(\lambda)=\sum_{i=1}^{m-1}\left(-2 \log U_{(i)}\right)=2 \sum_{i=1}^{m-1} \log \left(\frac{S_{m}}{S_{i}}\right)
$$

has $\chi_{2(m-1)}^{2}$. This completes the proof.

By Lemma 1 in the work of Seo and Kang [9] and Lemma 2.2, $Q_{2}(\lambda) /[2(m-2)]$ converges to one in probability as $m \longrightarrow \infty$, which leads to an equation $Q_{2}(\lambda)=2(m-2)$ that
Proof. By Wang et al. [8], a quantity based on the quantity (3) is given by has a unique solution for $\lambda$ because the argument of the log term in (16) can be written as

$$
1+\frac{\sum_{j=i+1}^{m}\left(R_{j}+1\right)\left(e^{\lambda X_{j: m: n}}-1\right) /\left(e^{\lambda X_{i: m: n}}-1\right)-\left[n-\sum_{j=1}^{i}\left(R_{j}+1\right)\right]}{\sum_{j=1}^{i}\left(R_{j}+1\right)\left(e^{\lambda X_{j: m: n}}-1\right) /\left(e^{\lambda X_{i: m: n}}-1\right)+\left[n-\sum_{j=1}^{i}\left(R_{j}+1\right)\right]}
$$

and the term $\left(e^{\lambda X_{j: m: n}}-1\right) /\left(e^{\lambda X_{i: m: n}}-1\right)$ in (19) is an increasing function of $\lambda$ (resp. decreasing) for $j>$ (resp. $<) i$. The unique solution is denoted as $\hat{\lambda}_{p}$. 
TABLE 2: Progressive Type-II censored tumor-free time data and corresponding censoring scheme.

\begin{tabular}{|c|c|c|c|c|c|c|c|c|c|c|c|c|c|c|c|c|c|}
\hline$i$ & 1 & 2 & 3 & 4 & 5 & 6 & 7 & 8 & 9 & 10 & 11 & 12 & 13 & 14 & 15 & 16 & 17 \\
\hline$X_{i: m: n}$ & 0.60 & 0.63 & 0.66 & 0.66 & 0.68 & 0.70 & 0.70 & 0.77 & 0.77 & 0.84 & 0.91 & 0.91 & 0.94 & 0.98 & 1.01 & 1.08 & 1.09 \\
\hline$R_{i}$ & 2 & 0 & 0 & 0 & 3 & 0 & 0 & 0 & 1 & 0 & 0 & 2 & 0 & 0 & 0 & 0 & 5 \\
\hline
\end{tabular}

TABLE 3: Estimates of $\lambda$ and $\theta$ for real data.

\begin{tabular}{lccccc}
\hline$\hat{\lambda}$ & $\hat{\lambda}_{p}$ & $\hat{\lambda}_{w l}$ & $\hat{\theta}(\hat{\lambda})$ & $\hat{\theta}_{p}\left(\hat{\lambda}_{p}\right)$ & 0.021 \\
\hline 5.549 & 5.362 & 5.448 & 0.019 & 0.021 \\
\hline
\end{tabular}

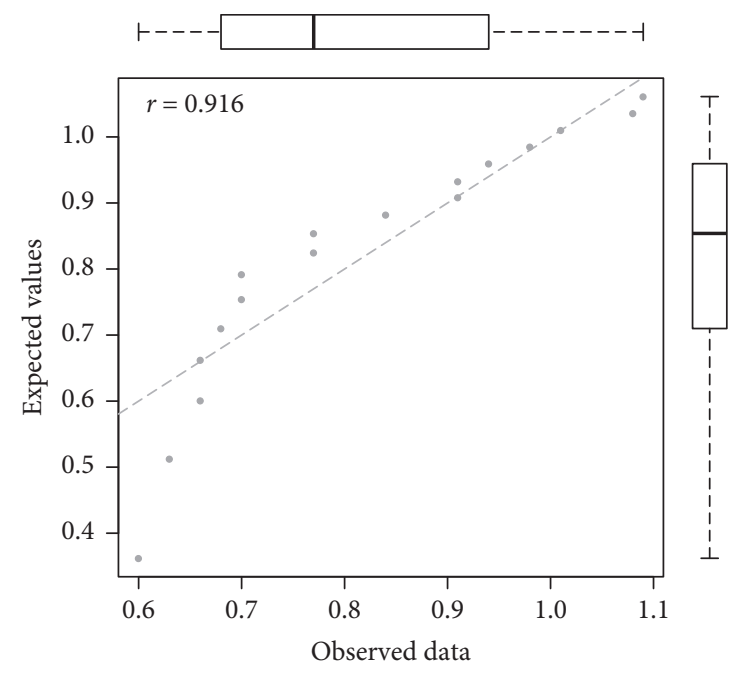

(a)

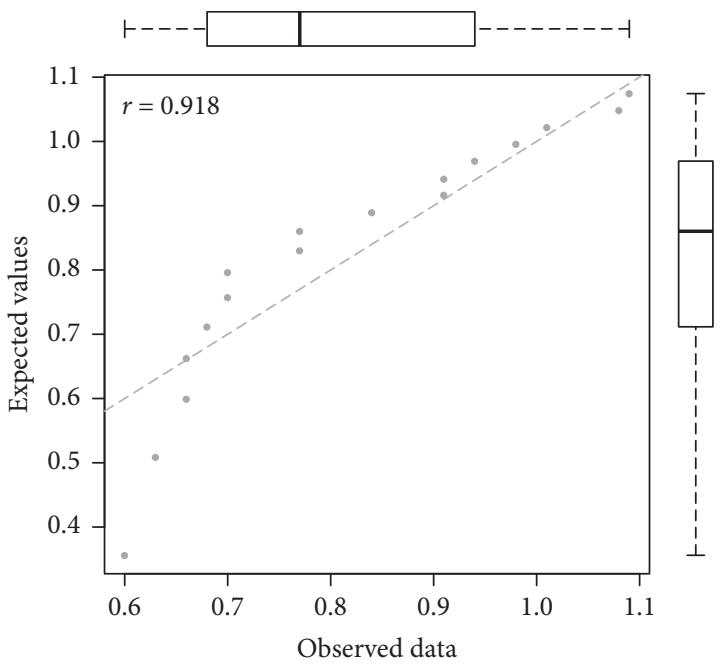

(b)

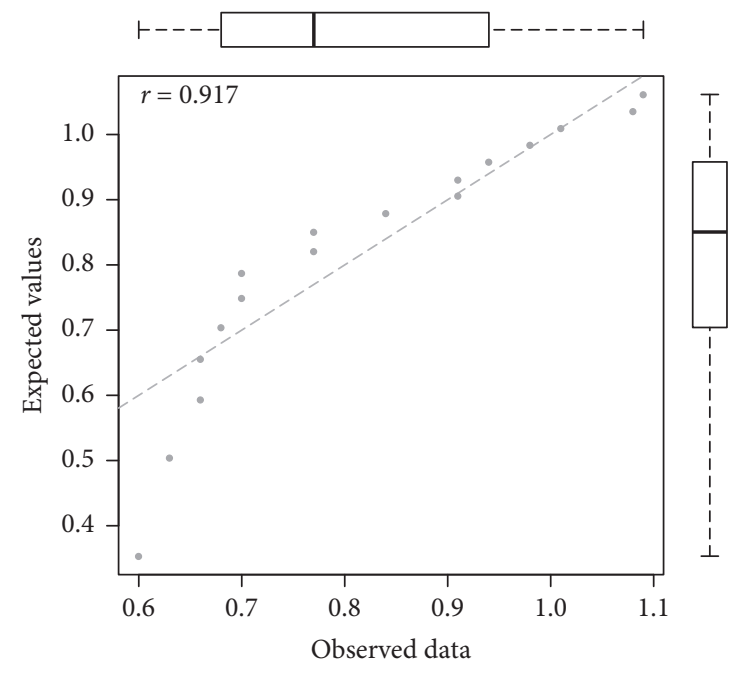

(c)

FIGURE 1: Box-scatter plots with (a) the MLEs $\hat{\lambda}$ and $\hat{\theta}(\hat{\lambda}) ;$ (b) the pivot-based estimators $\hat{\lambda}_{p}$ and $\hat{\theta}_{p}\left(\widehat{\lambda}_{p}\right) ;(\mathrm{c})$ the weighted least-estimators $\hat{\lambda}_{w l}$ and $\widehat{\theta}_{w l}\left(\widehat{\lambda}_{w l}\right)$. 


\section{Application}

This section assesses and compares the estimation methods provided in Section 2 through the Monte Carlo simulations and real data analysis.

3.1. Simulation Study. For evaluation and comparison, the mean squared errors (MSEs) and biases of the provided estimators are reported in Table 1. The progressive Type-II censored samples are generated from the Gompertz distribution with $\lambda=0.1$ and $\theta=0.5$ under the following scenarios

Scheme I: $R_{m}=n-m$ and $R_{i}=0$ for $i \neq m$

Scheme II: $R_{1}=n-m$ and $R_{i}=0$ for $i \neq 1$

Scheme III: $R_{1}=R_{m}=((n-m) / 2)$ and $R_{i}=0$ for $i \neq$ 1 and $m$

Scheme IV: $R_{m / 2}=n-m$ and $R_{i}=0$ for $i \neq(m / 2)$

by using the algorithm of Balakrishnan and Aggarwala [14]. All MSEs and biases are computed based on the generated 1,000 progressive Type-II censored datasets.

From Table 1 , it can be seen that the weighted leastsquare estimator $\widehat{\lambda}_{w l}$ is more efficient compared to the MLE $\hat{\lambda}$ in terms of the bias, but the pivot-based estimator $\widehat{\lambda}_{p}$ shows the best performance in terms of both the MSE and bias. Even for $\theta$, the pivot-based estimator $\widehat{\theta}_{p}\left(\widehat{\lambda}_{p}\right)$ generally shows better results compared to the MLE $\hat{\theta}(\widehat{\lambda})$ and the weighted least-square estimator $\widehat{\theta}_{w l}\left(\widehat{\lambda}_{w l}\right)$. Finally, MSEs of all estimators decreases as expected with the increasing number of failures $m$ for a fixed sample size.

3.2. Real Data. Chen [2] and Lee [17] analyzed a real data set that represents tumor-free time in days of 30 rats fed with unsaturated diet. For illustration purposes, a progressive Type-II censored data are generated from the tumor-free time data after dividing each data point by 100 . The censoring scheme and corresponding censored data are presented in Table 2. Prior to analysis, the goodness-of-fit test based on the first moment is first conducted. To avoid computational complex, the follow empirical first moment is employed:

$$
E\left(X_{i: m: n} ; \lambda, \theta\right)=\frac{1}{N} \sum_{j=1}^{N} X_{i: m: n}^{j}, \quad i=1, \ldots, m,
$$

where $X_{i: m: n}^{j}(j=1, \ldots, N)$ are samples from the marginal distribution $f_{X_{i: m: n}}(x ; \lambda, \theta)$. The first moment (3.1) is evaluated at all the estimators provided in Section 2. Figure 1 reports the results through the box-scatter plot and the correlation coefficient $(r)$ between the generated progressive Type-II censored data and the corresponding empirical first moments, which indicates that the assumption that the censored tumor-free time data have the Gompertz distribution which is reasonable for all estimates. The estimation results for $\lambda$ and $\theta$ are reported in Table 3, which shows that the weighted least-square and pivot-based estimators have values similar to those of the MLEs under the considered schemes.

\section{Conclusions}

This paper provides approaches based on the weighted regression framework and pivotal quantity to estimate unknown parameters of the Gompertz distribution with the PDF (1) under the progressive Type-II censoring scheme. The proposed methods are comparatively concise and easy to perceive compared with the existing methods such as the maximum likelihood method. In addition, it was proved that the pivot-based estimators are superior to the MLEs and weighted least-square estimators in terms of the MSE and bias. Although the result for the goodness-of-fit test does not have statistically significant differences, when considered with the simulation results, it is highly suggested the use of the estimation method based on the pivotal quantity for case where progressive Type-II censored data from the Gompertz distribution are observed.

\section{Data Availability}

The censored data used to support the findings of this study are included within the article.

\section{Conflicts of Interest}

The authors declare that they have no conflicts of interest.

\section{Acknowledgments}

This work was supported by the National Research Foundation of Korea (NRF) Grant Funded by the Korea Government (Ministry of Education) (no. 2019R1I1A3A01062838).

\section{References}

[1] B. Gompertz, "On the nature of the function expressive of the law of human mortality and on the new mode of determining the value of life contingencies," Philosophical Transactions of the Royal Society of London, vol. 115, pp. 513-580, 1825.

[2] Z. Chen, "Parameter estimation of the Gompertz population," Biometrical Journal, vol. 39, no. 1, pp. 117-124, 1997.

[3] S. J. Wu, C. T. Chang, and T. R. Tsai, "Point and interval estimations for the Gompertz distribution under progressive type-II censoring," Metron, vol. 61, no. 3, pp. 403-418, 2003.

[4] S. Dey, T. Kayal, and Y. M. Tripathi, "Evaluation and comparison of estimators in the Gompertz distribution," Annals of Data Science, vol. 5, no. 2, pp. 235-258, 2018.

[5] S. Dey, F. A. Moala, and D. Kumar, "Statistical properties and different methods of estimation of Gompertz distribution with application," Journal of Statistics and Management Systems, vol. 21, no. 5, pp. 839-876, 2018.

[6] F. A. Moala and S. Dey, "Objective and subjective prior distributions for the Gompertz distribution," Anais da Academia Brasileira de Ciências, vol. 90, no. 3, pp. 2643-2661, 2018.

[7] S. J. Wu, "Estimation of the two-parameter bathtub-shaped lifetime distribution with progressive censoring," Journal of Applied Statistics, vol. 35, no. 10, pp. 1139-1150, 2008.

[8] B. X. Wang, K. Yu, and M. C. Jones, "Inference under progressively type II right-censored sampling for certain lifetime distributions," Technometrics, vol. 52, no. 4, pp. 453-460, 2010. 
[9] J. I. Seo and S. B. Kang, "Pivotal inference for the scaled half logistic distribution based on progressively Type-II censored samples," Statistics \& Probability Letters, vol. 104, pp. 109-116, 2015.

[10] J. I. Seo and S. B. Kang, "Inference for the two-parameter halflogistic distribution using pivotal quantities under progressively type-II censoring schemes," Communications in Statistics-Simulation and Computation, vol. 46, no. 7, pp. 5462-5478, 2017.

[11] H. L. Lu and S. H. Tao, "The estimation of Pareto distribution by a weighted least square method," Quality \& Quantity, vol. 41, no. 6, pp. 913-926, 2007.

[12] J. I. Seo, S. B. Kang, and H. Y. Kim, "New approach for analysis of progressive type-II censored data from the Pareto distribution," Communications for Statistical Applications and Methods, vol. 25, no. 5, pp. 569-575, 2018.

[13] J. I. Seo, B. G. Seo, and S. B. Kang, "Estimation on a two-parameter Rayleigh distribution under the progressive type-II censoring scheme: comparative study," Communications for Statistical Applications and Methods, vol. 26, no. 2, pp. 91-102, 2019.

[14] N. Balakrishnan and R. Aggarwala, Progressive Censoring-Theory, Methods, and Applications, Birkhäuser, Boston, MA, USA, 2000.

[15] N. Balakrishnan and E. Caramer, The Art of Progressive Censoring, Springer, New York, NY, USA, 2014.

[16] A. F. Karr, Probability, Springer-Verlag, New York, NY, USA, 1993.

[17] E. T. Lee, Statistical Methods for Survival Data Analysis, Wiley, New York, NY, USA, 2nd edition, 1992. 